\title{
Qualität in der Weiterbildung: Aktueller Stand und Zukunft psychotherapeutischer Aus- und Weiterbildung
}

\author{
Jürgen Tripp ${ }^{a} \quad$ Ulrich Schweiger ${ }^{b} \quad$ Walter Ströhm $^{c} \quad$ Claudia Stromberg $^{d} \quad$ Kurt Quaschner $^{\mathrm{e}}$ \\ a Deutscher Fachverband für Verhaltenstherapie und Psychotherapeutische Praxis, Münster, Deutschland; \\ ${ }^{b}$ Klinik für Psychiatrie und Psychotherapie, Universität zu Lübeck, Lübeck, Deutschland; \\ ${ }^{c}$ Gesellschaft für Angewandte Psychologie und Verhaltensmedizin (APV), Münster, Deutschland; \\ dGesellschaft für Ausbildung in Psychotherapie (GAP), Frankfurt/M., Deutschland; \\ e Klinik für Kinder- und Jugendpsychiatrie, -psychosomatik und -psychotherapie am Universitätsklinikum Marburg und Gießen, \\ Standort Marburg, Marburg, Deutschland
}

\section{Schlüsselwörter}

Psychotherapieausbildung · Weiterbildung · Direktausbildung · Psychotherapeutische Kompetenzen

\section{Zusammenfassung}

Das Reformkonzept der psychotherapeutischen Direktausbildung, das eine Angleichung der Ausbildungsstruktur der Psychologischen Psychotherapeuten und Kinder- und Jugendlichenpsychotherapeuten an die anderer akademischer Heilberufe mit einem durch eine Approbationsordnung geregelten Studium, Staatsexamen und anschließender Weiterbildung beinhaltet, stößt zum Teil auf deutliche Kritik. Insbesondere wird kritisiert, dass in einer Weiterbildung analog zur Facharztausbildung die Qualität der jetzigen Psychotherapieausbildung nicht aufrechterhalten werden könne. Um diese Diskussion auf eine fundierte Basis zu stellen, legen wir dar, was aus unserer Sicht zur Qualität psychotherapeutischer Ausbildung beiträgt, und stellen empirische Befunde zur Qualität der Psychotherapieausbildung sowie zur ärztlichen Weiterbildung vor. Dargestellt werden außerdem Konsequenzen für eine zukünftige Weiterbildung nach einer Direktausbildung, welche notwendig erscheinen, um für eine hohe Qualität des Kompetenzerwerbs zu sorgen: Die Weiterbildung sollte eine Verbindung von praktischem Erfahrungserwerb unter Anleitung mit den bewährten bisherigen Ausbildungselementen wie Theorieworkshops und Selbsterfahrung ermöglichen. Es sollten verschiedene Versorgungssettings und Patientengruppen kennengelernt werden. Die bisherigen Ausbildungsinstitute sollten als Weiterbildungsinstitute weiterhin eine zentrale Rolle spielen und mit anderen Weiterbildungsstätten kooperieren. Der Kompetenzerwerb sollte beschrieben werden und überprüfbar sein und den Psychotherapeutenkammern käme eine wichtige Funktion als Aufsichtsund Anerkennungsinstanz zu. Damit diese Qualitätsmerkmale umgesetzt werden können, müssen die Weiterbildungsstrukturen finanziell ausreichend ausgestattet werden.

\section{Keywords}

Training in psychotherapy · Advanced professional training . Competence in psychotherapy

\section{Summary}

Quality in Advanced Professional Training: Current Status and Future of Advanced Professional Training in Psychotherapy

The reform concept of a 'Direktausbildung', which includes a mainstreaming of the structure of psychotherapy training to the structure of other academic health professions with an academic program that is regulated by national licensing regulations and followed by an advanced training program, is received with significant criticism in the professional community. The criticism in particular is that, in a development analogous to medical specialist training, the quality of the current psychotherapy training cannot be maintained. To put this discussion on a sound basis, we offer arguments on what in our view contributes to the quality of psychotherapeutic training and provide empirical evidence on the quality of psychotherapeutic training and advanced medical training. Shown are consequences for a future advanced training, which are deemed necessary to ensure a high quality of competence acquisition: The training should offer a combination of practical training on the job under supervision combined with established training elements, such as theory workshops and promotion of personal competencies (Selbsterfahrung). Various patient care settings and patient groups should be included in the training. The previous psychotherapy training institutes (Ausbildungsinstitute) should continue to play a central role as advanced training institutes (Weiterbildungsinstitute) and cooperate with other advanced training facilities. The acquisition of competence should be defined in detail and should be verifiable, and psychotherapist chambers would have an important function as a surveillance and accreditation institution. In order to implement these quality features, the training structures must be equipped with sufficient financial means.

\section{KARGER




\section{Einleitung}

Beim 25. Deutschen Psychotherapeutentag im November 2014 sprach sich eine breite Mehrheit der Delegierten für eine umfassende Reform der Psychotherapieausbildung aus. Das Reformmodell, das auf Basis dieses Beschlusses von der Bundespsychotherapeutenkammer vertreten wird und gemeinhin unter dem Schlagwort «Direktausbildung» bekannt ist, sieht im Wesentlichen vor, dass die Approbation als Psychotherapeut durch ein Hochschulstudium mit wissenschaftlicher und praktischer Ausrichtung erworben wird, ohne dass dabei schon eine Festlegung auf ein Verfahren oder einen Altersschwerpunkt erfolgt. Die Fachkunde könnte dann in einer auf einen Altersschwerpunkt und ein Verfahren spezialisierten Weiterbildung nach dem Studium erworben werden. Dieser Vorschlag würde darauf hinauslaufen, die Psychotherapeutenausbildung an die Struktur der Aus- und Weiterbildung in anderen akademischen Heilberufen anzugleichen [vgl. auch Lubisch, 2012; Rief et al., 2012a; Rief et al., 2012b; Körner, 2013]. Diese Beschäftigung mit einer Direktausbildung löste jedoch auch massive Kritik von verschiedenen Akteuren aus, die zum Teil alternative Reformmodelle vorschlagen [z.B. Fliegel, 2013; Gleiniger, 2013; Sulz, 2013].

Von Ströhm et al. [2013; vgl. auch Tripp, 2013] wurde ein erster Vorschlag zur möglichen Umsetzung und Gestaltung einer Weiterbildung nach einer solchen Direktausbildung in Psychotherapie vorgelegt, in dem die Konsequenzen der Umsetzung der gesetzlichen Vorschriften für heilberufliche Weiterbildung durchgespielt werden. Eines der Hauptargumente gegen dieses Konzept einer Weiterbildung zur Erlangung der Fachkunde nach einem Direktstudium ist, dass die hohe Qualität der jetzigen Psychotherapieausbildung im Rahmen einer Weiterbildung nach dem Vorbild der ärztlichen Facharztausbildung nicht umgesetzt werden könne [Sulz und Hoenes, 2014]. Zudem wird häufig die Befürchtung geäußert, dass die Weiterbildung dann hauptsächlich in Kliniken und eventuell noch in Praxen von niedergelassenen Psychotherapeuten stattfinden würde und die bisherigen Ausbildungsinstitute kaum eine oder gar keine Rolle mehr spielen würden, was auch der Qualität abträglich sei, da die Ausbildungsinstitute ein Garant der Qualität in der Ausbildung seien. Die Qualität der ärztlichen Psychotherapiequalifikation im Rahmen der Facharztweiterbildung wird als Negativbeispiel dargestellt, das nicht durch die Anpassung der psychotherapeutischen Aus- und Weiterbildungsstruktur an das ärztliche Modell übernommen werden sollte [vgl. Sulz und Hoenes, 2014].

Wir möchten im Folgenden diese Diskussion auf eine fundierte Basis stellen, indem wir zunächst darstellen, was die Qualität einer guten Psychotherapieausbildung im Allgemeinen und der jetzigen Psychotherapieausbildung ausmacht und welche strukturellen Rahmenbedingungen zu guter Qualität in der Psychotherapieausbildung beitragen. Dann werden wir auf die Qualität der ärztlichen Weiterbildung eingehen sowie auf die aktuelle Diskussion in der Ärzteschaft über Qualitätsprobleme in der Weiterbildung und Reformvorschläge für die ärztliche Weiterbildung. Auf dieser Basis diskutieren wir dann Perspektiven für das Erlernen von Psychothe- rapie in einer Weiterbildung auf hohem Qualitätsniveau. Wir befassen uns mit der Frage, welche Rahmenbedingungen hierfür notwendig sind, und leiten daraus erste Vorschläge für eine mögliche psychotherapeutische Gebietsweiterbildung zur Erlangung der Fachkunde nach einem Direktstudium ab.

\section{Qualität der jetzigen Psychotherapieausbildung}

Hohe Qualität in der psychotherapeutischen Ausbildung wird vor allem dadurch erreicht, dass sowohl theoretische Kenntnisse als auch praktische Fertigkeiten vermittelt werden und dass theoretische Kenntnisse und praktisches Handeln aufeinander bezogen sind [Sachse et al., 2014]. Die Erlangung von psychotherapeutischer Kompetenz setzt ein längerfristiges Training unter Anleitung von Experten voraus, bei dem im Sinne einer «Deliberate Practice» bzw. reflektierter Praxis [Skovholt et al., 1997; Ericsson, 2008; Sachse et al., 2014] die Inhalte handlungsorientiert vermittelt und Erfahrungen im Praxisfeld permanent durch Rückmeldungen von Experten begleitet werden, sodass das praktische Handeln reflektiert und optimiert werden kann.

Neben der Vermittlung von Faktenwissen, Begründungswissen und praktischen Handlungskompetenzen sollte die psychotherapeutische Ausbildung auch personale Kompetenzen fördern, wie z.B. die Fähigkeit zur Empathie, zur Rollenübernahme und zur Regulation von Nähe und Distanz, sowie Fähigkeiten zur Selbstreflexion vor dem Hintergrund der eigenen Biografie und zur Wahrnehmung und Regulation von eigenen Affekten und Impulsen. All dies trägt auch dazu bei, eine professionelle psychotherapeutische Haltung zu entwickeln [Bundespsychotherapeutenkammer, 2014; Richter, 2013].

Den Kontext einer nicht zu großen und nach Möglichkeit konstanten Lerngruppe halten wir für diesen Prozess der Kompetenzentwicklung ebenfalls für bedeutsam. Dies bietet die Möglichkeit, sehr direkt auch an den Erfahrungen von Kollegen teilzuhaben sowie im informellen Austausch viel voneinander zu lernen und nicht zuletzt auch soziale Unterstützung bei der Bewältigung von schwierigen Herausforderungen und belastenden oder irritierenden Erfahrungen zu erhalten.

In der jetzigen Psychotherapieausbildung werden diese Erkenntnisse konzeptionell in der Theorievermittlung, der Praktischen Ausbildung unter Supervision und der Selbsterfahrung umgesetzt. In den Theorieveranstaltungen, die in der Regel in Form von Workshops in Gruppen von etwa 10-20 Teilnehmern stattfinden, wird einerseits Wissen vermittelt, es werden aber auch praktische Fähigkeiten trainiert, z.B. durch Live- oder Video-Demonstrationen von Therapiesituationen und durch Übungen wie z.B. Rollenspiele mit Feedback durch Teilnehmer und Dozenten. In diesem Lehrformat sind somit Theorie und Praxis in hohem Maße verknüpft und unmittelbar aufeinander bezogen und der oben genannte Gruppenaspekt kommt zum Tragen. Der Anspruch der reflektierten Praxis wird auch vor allem in den Ausbildungstherapien unter Supervision erfüllt, bei der das Handeln im relevanten Kontext einem regelmäßigen Feedback durch Supervisoren als Exper- 
ten unterliegt. Die Selbstreflektion erfolgt in der Gruppen- und/ oder Einzelselbsterfahrung.

Doch wie sieht es mit der tatsächlichen Umsetzung dieses Qualitätsanspruchs in der Praxis der Psychotherapieausbildung aus?

Mit Bezug auf das Forschungsgutachten zur Psychotherapieausbildung [Strauß et al., 2009] wird von einigen Fachgesellschaften und Vertretern von Ausbildungsinstituten immer wieder auf die hohe Qualität der jetzigen Psychotherapieausbildung hingewiesen. Wenn man sich die konkreten Aussagen des Forschungsgutachtens zur Qualität der Ausbildung ansieht, fällt auf, dass gar nicht so viele der dort erhobenen Daten eine empirische Aussage über die tatsächliche Qualität zulassen. Zumindest wurde Qualität nicht in dem Sinne erfasst, dass die Entwicklung von psychotherapeutischer Kompetenz als «Outcome» der Ausbildung erhoben wurde. Die Bewertung der Qualität basiert hier im Wesentlichen auf Selbstauskünften von Ausbildungsteilnehmern und den anderen an der Ausbildung beteiligten Personengruppen sowie Expertenmeinungen.

Im Forschungsgutachten bewerten die zum Befragungszeitpunkt in Ausbildung befindlichen Teilnehmer die Zufriedenheit mit verschiedenen Aspekten der Ausbildung im Mittel mit 3,38 auf einer 5-stufigen Skala, was einer Bewertung zwischen den Skalenpunkten «mittel» und «ziemlich zufrieden» entspricht. (Anmerkung: Die Mittelwertbildung über alle Zufriedenheitsskalen hinweg wurde nicht im Original vorgenommen, sondern hier der Übersicht halber durch die Verfasser berechnet.) Die ehemaligen Ausbildungsteilnehmer waren mit einem Mittelwert von 3,08 im Durchschnitt aller Zufriedenheitsskalen nur mittelmäßig zufrieden. Die Aspekte mit der höchsten Zufriedenheit waren die Atmosphäre am Ausbildungsinstitut und die Erreichbarkeit von Ansprechpartnern. Die geringste Zufriedenheit bestand bei den Mitsprachemöglichkeiten der Teilnehmer und dem Preis-LeistungsVerhältnis des Angebotes [Strauß et al., 2009: S. 84-85]. Bei der Frage nach der Nützlichkeit für die psychotherapeutische Kompetenzentwicklung wurden von den Ausbildungsteilnehmern die Praktische Ausbildung, die Supervision und die Selbsterfahrung mit jeweils hohen bis sehr hohen Zustimmungswerten als am nützlichsten für die psychotherapeutische Kompetenzentwicklung bewertet. Die Praktische Tätigkeit, Prüfungsvorbereitung, Kleingruppen und die freie Spitze werden im Vergleich dazu als am wenigsten nützlich bewertet. Dabei liegt der Mittelwert für die Praktische Tätigkeit hier auch noch bei 3,76 auf einer 5-stufigen Skala. Die durchschnittliche Bewertung rangiert also auch noch zwischen «mittel» und «ziemlich nützlich». Von den ehemaligen Ausbildungsteilnehmern wurden ebenfalls die Praktische Ausbildung und die Supervision als sehr nützlich für die Kompetenzentwicklung bewertet. Die Theorie und die Selbsterfahrung wurden hingegen im Vergleich zur Befragung der derzeit in Ausbildung befindlichen Teilnehmer als etwas weniger nützlich eingeschätzt [Strauß et al., 2009: S. 137-140].

Bei der Bewertung der Praktischen Tätigkeit zeigten sich gravierende Qualitätsmängel. So wurde deutlich, dass in vielen Einrichtungen der Praktischen Tätigkeit keine angemessene Einarbeitung und Anleitung erfolgt und dass die Ausbildungsteilnehmer häufig schon psychotherapeutische Tätigkeiten übernehmen, ohne dabei angemessene Supervision zu erhalten und ohne über eine heilkundliche Erlaubnis hierfür zu verfügen. Als wesentliche Ursachen für diese Mängel werden ein Struktur- und Integrationsmangel aufgrund der Externalität der meisten Einrichtungen der Praktischen Tätigkeit und die begrenzten Steuerungsmöglichkeiten und -intentionen der meisten Ausbildungsstätten gesehen [Strauß et al., 2009: S. 338-342].

Ein weiterer problematischer Aspekt der jetzigen Psychotherapieausbildung ist nach unserer Auffassung, dass das zugangsqualifizierende Studium komplett ohne obligatorische Erfahrungen mit ersten Patientenkontakten absolviert werden kann und dass auch in der Ausbildung unter Umständen nur ein recht eingeschränktes Patientenspektrum kennengelernt wird. So kann man z.B. die ganze Ausbildung absolvieren und dabei nur 2 verschiedene Behandlungseinrichtungen kennenlernen (z.B. eine Klinik, in der auf einer Station die Praktische Tätigkeit I und II (PT I \& II) absolviert werden, und die Ambulanz des Ausbildungsinstitutes). Dies spiegelt sich auch in Daten aus dem Forschungsgutachten. So werden z.B. Patienten mit Schizophrenie nur von $60 \%$, Patienten mit Suchterkrankungen nur von 57\% und Patienten mit Essstörungen nur von 52\% der Befragten in der Praktischen Tätigkeit kennengelernt (die Prozentzahlen für die Praktische Ausbildung liegen für die Behandlung dieser Störungen noch niedriger) [Strauß et al., 2009: S. 160]. Das heißt, es ist sehr wahrscheinlich, dass viele Psychotherapeuten ihre Ausbildung absolvieren und dabei nur ein eingeschränktes Patientenspektrum kennenlernen. Insbesondere für die Behandlung von sehr schweren oder nicht ganz so häufig vorkommendenden psychischen Erkrankungen ist der Erfahrungserwerb in der Ausbildung somit unvollständig.

Zusammenfassend kann man also feststellen, dass die Zufriedenheit der Ausbildungsteilnehmer zwar nicht schlecht ist, dass es aber auch einen belangvollen Anteil an Teilnehmern gibt, die mit einzelnen Aspekten nicht zufrieden sind, und dass die Zufriedenheit bei einer durchschnittlich nur etwas mehr als mittelmäßigen Bewertung durchaus noch steigerbar erscheint. In Bezug auf die Kompetenzentwicklung werden mit der Praktischen Ausbildung unter Supervision, der Selbsterfahrung und (mit Abstrichen) der Theorie insbesondere die Ausbildungsbestandteile als nützlich eingeschätzt, die in hohem Maße den Anspruch an eine reflektierte Praxis umsetzen. Bei der Praktischen Tätigkeit scheint es Probleme mit der Qualität zu geben, weil hier der Anspruch einer reflektierten Praxis häufig nicht umgesetzt wird. Dies scheint unter anderem daran zu liegen, dass die Modalitäten der Anleitung und Aufsicht und somit der Rückmeldung und Reflektion der Tätigkeit nicht so klar definiert sind wie bei der Praktischen Ausbildung, dass die Verantwortlichkeit für die Anleitung und Aufsicht nicht klar benannt ist und dass aufgrund der Externalität der kooperierenden Einrichtungen die dort gemachten praktischen Erfahrungen nicht so gut auf die anderen Inhalte und Elemente der Ausbildung bezogen sind. Neben diesen Aspekten dürfte natürlich noch die Diskrepanz zwischen Qualifikation und erbrachter Leistung der Ausbildungsteilnehmer einerseits und der dafür erfahrenen monetären und persönlichen Wertschätzung («Praktikantenstatus») anderer- 
seits einen Einfluss auf die eher negative Bewertung der Praktischen Tätigkeit und die häufig geäußerte Forderung nach deren Verkürzung haben.

Wenn man sich anschaut, welche strukturellen Merkmale zur Qualität der Psychotherapieausbildung beitragen, sind zunächst natürlich die Bestimmungen zu Ausbildungsbestandteilen und Mindestanforderungen des Psychotherapeutengesetzes und der Ausbildungs- und Prüfungsverordnung relevant ( $\$ 6$ PsychThG). Hier werden als Träger der Ausbildung die staatlich anerkannten Ausbildungsstätten definiert. Diese müssen einige Kriterien erfüllen. Es müssen ausreichend Patienten für die Ausbildung zur Verfügung stehen und diese müssen in Richtlinienverfahren behandelt werden. Die Ausbildungsstätten müssen über eine angemessen Ausstattung verfügen und es muss Fachpersonal (Psychologische Psychotherapeuten (PP), Kinder- und Jugendlichenpsychotherapeuten (KJP), Ärzte) in ausreichender Zahl zur Anleitung zur Verfügung stehen. Die Ausbildung muss nach Ausbildungsplänen durchgeführt werden, die sich am Gegenstandskatalog der Ausbildungs- und Prüfungsverordnung orientieren. Die Ausbildungsstätten müssen gewährleisten, dass die Praktische Tätigkeit unter Anleitung und Aufsicht stattfindet, und sie müssen die theoretische und praktische Ausbildung anbieten.

Es wird hier also zunächst von einer Einrichtung ausgegangen, die als Ausbildungsstätte alle diese Anforderungen erfüllt und die komplette Ausbildung gewährleistet («Ausbildung aus einer Hand»). In Satz 3 des $\$ 6$ PsychThG wird dann jedoch die Möglichkeit eingeräumt, dass die Ausbildungsstätte, wenn sie die geforderte Praktische Tätigkeit, die theoretische Ausbildung oder die praktische Ausbildung nicht vollständig durchführen kann, sicherstellen muss, dass diese Aufgaben von einer anderen geeigneten Einrichtung übernommen werden.

In der Praxis hat es sich als Regelfall etabliert, dass Institute, die über eine Ausbildungsambulanz verfügen, die Ausbildung anbieten. Die Praktische Tätigkeit wird von ihnen jedoch nicht angeboten, sondern findet in kooperierenden Einrichtungen (zumeist Kliniken) statt. Gelegentlich werden auch Teile der praktischen Ausbildung an kooperierende externe Einrichtungen delegiert (Lehrpraxen). Kaum bekannt ist jedoch der Fall, dass eine Ausbildungsstätte die theoretische Ausbildung an eine andere Einrichtung delegiert. Dass sich das Modell der Ausbildung an einem Institut mit Ambulanz und Kooperation mit Kliniken weitgehend durchgesetzt hat, liegt wohl nicht in erster Linie an den gesetzlichen Bestimmungen, sondern an der Tradition der Psychotherapieausbildung an privaten Instituten und den hier bereits vor dem Psychotherapeutengesetz schon vorhandenen Strukturen.

Als Prinzip wird jedoch grundsätzlich deutlich, dass die Ausbildung an einer Einrichtung stattfindet, die für den strukturierten Ablauf der gesamten Ausbildung verantwortlich ist und auch die Verantwortung für die Durchführung einzelner Ausbildungsbestandteile in externen Einrichtungen hat.

Für die Kontrolle der Einhaltung der Bestimmungen des Psychotherapeutengesetzes und der Ausbildungs- und Prüfungsverordnung sind die Landesprüfungsämter für Gesundheitsberufe der Bundesländer zuständig. Obwohl es sich um bundesweit ein- heitlich gültige Bestimmungen handelt, gibt es in der Auslegung und Anerkennungspraxis zum Teil deutliche Differenzen zwischen den einzelnen Landesprüfungsämtern. So wird im Forschungsgutachten festgestellt, dass nicht alle Landesprüfungsämter die Festlegung der Ausbildungsstätten auf eine Vertiefungsrichtung verlangen, die Vorgaben bezüglich einzelner Ausbildungsbausteine (z.B. Selbsterfahrung) nicht einheitlich sind, nicht alle Landesprüfungsämter die Qualifikation von Supervisoren und Selbsterfahrungsleitern überprüfen und es deutliche Unterschiede zwischen den Landesprüfungsämtern bei der Anerkennung von Zugangsberechtigungen zur Psychotherapieausbildung gibt. Die Landesprüfungsämter beklagen in der Befragung durch die Forschergruppe Personalknappheit und eine hohe Arbeitsbelastung [Strauß et al., 2009: S. 76-78].

Neben dieser offensichtlich nur beschränkt als wirkliche Qualitätskontrolle wirksamen Überprüfung durch die Aufsichtsbehörden sind 85\% der Ausbildungsinstitute in Dachgesellschaften oder Verbänden organisiert und 70\% davon geben an, dass es in diesen Dachgesellschaften verbindliche Regularien für die Ausbildungsinstitute gibt [Strauß et al., 2009: S. 82]. Hier besteht also auch eine quasi freiwillige Aufsicht und Qualitätskontrolle durch den Berufsstand selbst, die wohl daher rührt, dass viele Vertreter psychotherapeutischer Verfahren ein Interesse an einer Nachwuchsbildung auf einem hohen Qualitätsniveau in ihrem jeweiligen Verfahren haben.

Zusammenfassend lässt sich feststellen, dass der Qualitätsanspruch einer reflektierten Praxis durch die Ausbildungsinstitute in der praktischen Ausbildung, der Theorievermittlung und der Selbsterfahrung in hohem Maße umgesetzt werden kann [Strauß et al., 2009; Weck, 2013]. Qualitätsprobleme gibt es mit der Praktischen Tätigkeit bei dem Teil der Ausbildung, der strukturell am schlechtesten in die Gesamtausbildung integriert ist und nur eingeschränkt einer Kontrolle durch die Ausbildungsinstitute und somit durch den eigenen Berufsstand der Psychotherapeuten unterliegt. Das praktische Erfahrungsspektrum ist häufig auf die typische ambulante Behandlungssituation und einen eingeschränkten Ausschnitt der stationären Behandlung beschränkt. Die Konzeption einer «Ausbildung aus einer Hand» sorgt, soweit sie umgesetzt werden kann, für eine Koordinierung und inhaltliche Abstimmung der verschiedenen Ausbildungselemente.

\section{Qualität der ärztlichen Weiterbildung und Reformdiskussion zur ärztlichen Weiterbildung}

In der Ärzteschaft wird in jüngster Zeit intensiv über eine Reform der ärztlichen Weiterbildung diskutiert. Anlass hierfür waren die Ergebnisse der Evaluation der ärztlichen Weiterbildung in den Jahren 2009 und 2011, der mittlerweile breit getragene Wunsch nach einer stärkeren Einbeziehung des ambulanten Sektors in die Weiterbildung sowie Probleme der jetzigen Weiterbildung wie z.B. inhaltliche Überfrachtung, überhöhte Richtzahlen und die hohe Arbeitsbelastung der Ärzte in Weiterbildung [Bundesärztekammer, 2013; David et al., 2013; Bartmann, 2013]. 
Bei der Evaluation der Weiterbildung durch die Bundesärztekammer [Bundesärztekammer und Consumer Behaviour Group ETH Zürich, 2011] zeigte sich in der globalen Beurteilung der Weiterbildung für alle Fachrichtungen eine mittlere bis gute Zufriedenheit mit einem Mittelwert von 2,4 auf einer Schulnotenskala (1-6), wobei 18,4\% der Befragten die Weiterbildung insgesamt nur «ausreichend» oder schlechter bewerteten. Die Bewertung der Vermittlung von Fachkompetenz, die Lernkultur, die Führungskultur, die Kultur zur Fehlervermeidung, die Entscheidungskultur und die Betriebskultur entsprechen weitestgehend der Globalbewertung. Nur die Bewertung der Evidenzbasierung ( Wissenschaftlich begründete Medizin») fällt hinter dem Globalurteil zurück. Hier gaben $41,5 \%$ die Note «ausreichend» oder eine noch schlechtere Note.

In der Subgruppenauswertung für die Fachrichtung Psychiatrie und Psychotherapie entsprechen die Ergebnisse der Evaluation im Wesentlichen den Gesamtergebnissen für alle Fachrichtungen [Bundesärztekammer, 2011a]. Bei der Subgruppe der Fachrichtung Psychosomatische Medizin und Psychotherapie ist die Globalbewertung mit 2,0 etwas besser als in der Gesamtauswertung über alle Fachgruppen. Dies zeigt sich auch in etwas besseren Bewertungen auf den Skalen «Vermittlung von Fachkompetenz», «Lernkultur» und «Führungskultur» [Bundesärztekammer, 2011b].

Die Weiterbildung im ambulanten Bereich wurde über alle Skalen hinweg besser eingeschätzt als die Weiterbildung im stationären Bereich [Bundesärztekammer und Consumer Behaviour Group ETH Zürich, 2011].

$\mathrm{Zu}$ den weiteren Fragen geben $42 \%$ der Weiterbildungsassistenten an, dass sie keinen strukturierten Weiterbildungsplan erhalten haben, und 32,7\% geben an, dass keine konkreten Weiterbildungsziele/Lernziele mit ihnen vereinbart wurden, obwohl diese Maßnahmen der Qualitätssicherung in den Weiterbildungsordnungen vorgeschrieben sind. Die Anleitung der Weiterbildungsassistenten in der praktischen Weiterbildung erfolgt in der Regel durch Oberärzte und Fachärzte (73,7\%). Nur 14,3\% werden direkt vom Chefarzt angeleitet (der in der Regel der Weiterbildungsbefugte sein dürfte). 6,4\% geben sogar an, dass sie in der Regel von erfahrenen Assistenzärzten angeleitet werden.

Externe Fort- oder Weiterbildungsmaßnahmen werden von $83,6 \%$ der Befragten regelmäßig besucht. Es scheint also weit verbreitet zu sein, dass in der ärztlichen Weiterbildung nicht mehr alle notwendigen Inhalte von der Weiterbildungsstätte selbst vermittelt werden können [Bundesärztekammer und Consumer Behaviour Group ETH Zürich, 2011].

In einer spezifischen Auswertung für die Fachrichtungen Kinder- und Jugendpsychiatrie, Psychiatrie und Psychosomatische Medizin gaben 99\% der Weiterbildungsbefugten an, den Weiterbildungsassistenten den Besuch externer Fort- und Weiterbildungen finanziell und/oder durch Freistellung zu ermöglichen. 69,3\% der Weiterbildungsassistenten gaben an, dass die Kosten für externe Weiterbildung teilweise vom Arbeitgeber übernommen werden und bei $17,5 \%$ gab es eine volle Kostenübernahme des Arbeitgebers. Hier zeigt sich, dass der Anspruch, dass die Weiterbildung den Assistenten nichts kosten sollte, in diesen Fachgebieten nur teilweise umgesetzt werden kann und 82,5\% der Befragten Kosten für externe Weiterbildungsmaßnahmen teilweise oder ganz selbst tragen müssen [Bundesärztekammer, 2011c]. Dies könnte so interpretiert werden, dass in den Fachgebieten, in denen Psychotherapie Bestandteil der Weiterbildung ist, Elemente dieser Qualifikation (z.B. Seminare, Selbsterfahrung) häufig über externe Angebote erbracht werden und für diese dann auch ein Finanzierungsbedarf besteht, der nur zum Teil vom Arbeitgeber getragen wird.

Zur Arbeitsbelastung geben 90,5\% an, dass Überstunden oder Mehrarbeit anfallen. Diese werden nur bei 58,6\% vollständig dokumentiert, bei $13,5 \%$ gar nicht und bei 38,8\% nur teilweise durch Geld oder Freizeitausgleich ausgeglichen. Bei der Frage, ob die Weiterbildung in der Arbeitszeit zur vollen Zufriedenheit erfüllt werden kann, äußerten sich $66 \%$ als unzufrieden.

Noch kritischer als in der Evaluation der Weiterbildung der Bundesärztekammer fallen die Ergebnisse einer Befragung des Marburger Bundes unter den in Facharztweiterbildung befindlichen Mitgliedern im Rahmen des MB-Monitors aus [Marburger Bund, 2014]. An der als repräsentativ bewerteten Onlinebefragung beteiligten sich 1118 Weiterbildungsassistenten, was einer Rücklaufquote von $10,36 \%$ entspricht.

Auch hier zeigte sich, dass nur wenige Assistenten direkt vom Chefarzt angeleitet werden (3\%). Die meisten (71\%) wurden von Ober- oder Fachärzten angeleitet. Der Anteil derer, die von Kollegen angeleitet wurden, die sich selbst in Weiterbildung befinden, lag mit 23\% jedoch deutlich höher als in der Evaluation der Bundesärztekammer.

Fast zwei Drittel der Befragten gaben an, dass sie die geforderten Weiterbildungsinhalte eher nicht (51\%) oder gar nicht (12\%) während der alltäglichen klinischen Arbeit vermittelt bekommen. Von 58\% werde erwartet, dass sie vorgeschriebene Weiterbildungsinhalte außerhalb der regulären Arbeitszeit absolvieren. Die Autoren des MB-Monitors kommen aufgrund dieser Ergebnisse zu folgendem Schluss: «Die hohe Arbeitsbelastung und enge Taktung im Klinikbetrieb lässt zu wenig Raum für die Weiterbildung im normalen Tagesablauf» [Marburger Bund, 2014: S. 3].

Bezüglich der Umsetzung der in den Weiterbildungsordnungen vorgesehenen Instrumente zur Qualitätssicherung zeigen sich auch in der Umfrage des Marburger Bundes noch drastischere Ergebnisse als in der Evaluation der Bundesärztekammer. So geben $85 \%$ an, dass ihnen kein strukturierter Weiterbildungsplan ausgehändigt worden sei. Und selbst wenn ein solcher Plan ausgehändigt worden sei, geben $57 \%$ an, dass er meist nicht eingehalten werde. Eine regelmäßige Rückmeldung zum Weiterbildungsstand, wie sie in Form eines jährlichen Weiterbildungsgespräches mit dem Weiterbildungsbefugten vorgesehen ist, findet nach Angabe von 47\% der Befragten überhaupt nicht statt, und auch der Einsatz der von den Ärztekammern zur Verfügung gestellten Logbücher zur Dokumentation des Weiterbildungsstandes wird von $67 \%$ verneint.

Nur 47\% der Befragten würden ihre Weiterbildungsstätte anderen zur Absolvierung der Weiterbildung empfehlen, die restlichen $53 \%$ hingegen nicht.

Die Ergebnisse der Evaluation der Weiterbildung der Bundesärztekammer und des MB-Monitors zeigen im Wesentlichen auf, 
dass die Qualität der ärztlichen Weiterbildung zum einen dadurch gefährdet ist, dass die Weiterbildung aufgrund von hoher Arbeitsbelastung in der Patientenversorgung zu kurz kommt, und zum anderen dadurch, dass Qualitätssicherungsinstrumente wie strukturierte Weiterbildungspläne, Weiterbildungsgespräche und Logbücher häufig nicht den Vorschriften gemäß eingesetzt werden.

Als weiteres Manko der ärztlichen Weiterbildung wird zunehmend beklagt, dass die Weiterbildung vorwiegend stationär und zu wenig im ambulanten Rahmen stattfinde [KBV, 2014]. Aufgrund der Tendenz zur Verlagerung von vielen Leistungen aus dem stationären in den ambulanten Bereich könne durch eine nur im Krankenhaus angesiedelte Weiterbildung nicht mehr alles gelernt werden, was für die spätere fachärztliche Tätigkeit relevant sei.

Daraus ergibt sich dann jedoch auch die Notwendigkeit, den Ablauf der Weiterbildung stärker zu koordinieren, wenn diese nicht mehr nur an einem Krankenhaus stattfindet, sondern verschiedene Einrichtungen in unterschiedlichen Versorgungssektoren umfasst. Hierzu wurden auf dem Deutschen Ärztetag die Entwicklung von strukturierten Weiterbildungsplänen und die Ermöglichung von Verbund-Weiterbildungen bzw. geplanter Rotation über verschiedene Einrichtungen vorgeschlagen [Bartmann, 2013]. Des Weiteren wurde zur Reform der Weiterbildungsordnung vorgeschlagen, dass die Weiterbildungsordnung entschlackt und überhöhte Richtzahlen abgebaut werden sollten. Die Weiterbildungsbefugten sollen über «Train-the-Trainer»-Programme didaktisch qualifiziert werden und es soll ein Tutorensystem eingeführt werden, bei dem den Weiterbildungsassistenten Tutoren (wohl Fach- oder Oberärzte der Weiterbildungsstätte) zugeordnet werden, die sie im klinischen Alltag anleiten und unterstützen. Auch soll der Arztberuf dadurch attraktiver gemacht werden, dass Arbeitszeitmodelle und Weiterbildungsbestimmungen familienfreundlicher gestaltet werden sollen.

Eine weitere Zielsetzung in der Reformdiskussion um die ärztliche Weiterbildung besteht darin, die Weiterbildung zukünftig kompetenzbasiert auszurichten, ähnlich wie es beim Medizinstudium schon mit dem Nationalen Kompetenzbasierten Lernzielkatalog Medizin (NKLM) erfolgt. Das bedeutet, dass statt einer kleinteiligen Aufzählung von Inhalten und starren Definition von Richtzahlen für medizinische Prozeduren Kompetenzblöcke definiert werden sollen. Die Kompetenz eines Facharztes setzt sich demnach aus mehreren Kompetenzblöcken zusammen, für die Kompetenzen auf 4 Kompetenzebenen beschrieben werden können. Die 1. Kompetenzebene beschreibt die fachlichen Grundlagen, die mit dem Studium erreicht und somit nicht Gegenstand der Weiterbildung sind. Die weiteren Kompetenzebenen beschreiben zunehmend vertiefte Kenntnisse, Erfahrungen und Fertigkeiten im Fachgebiet, die auf der höchsten Kompetenzebene dann selbstständig und routinemäßig durchgeführt werden können.

Noch einen Schritt weiter in diese Richtung geht die Gesellschaft für Medizinische Ausbildung (GMA), die in einer Veröffentlichung [Berberat et al., 2013] die Orientierung am Konzept der Anvertraubaren Professionellen Tätigkeiten (APT) vorschlägt. Auf Basis der definierten ärztlichen Kompetenzen sollen professionelle Tätigkeiten beschrieben werden, die die Kompetenzen widerspie- geln. Für diese Tätigkeiten werden die folgenden Leistungsniveaustufen beschrieben [Berberat et al., 2013: S. 14]:

- Der Arzt in Weiterbildung ist nicht in der Lage, die ärztliche Tätigkeit durchzuführen.

- Der Arzt in Weiterbildung kann die ärztliche Tätigkeit unter enger Begleitung durchführen.

- Der Arzt in Weiterbildung kann die ärztliche Tätigkeit durchführen, wenn er Unterstützung anfordern kann.

- Der Arzt in Weiterbildung kann die ärztliche Tätigkeit selbstständig durchführen.

- Der Arzt in Weiterbildung kann andere bei der Durchführung der ärztlichen Tätigkeit betreuen.

Die APT sind hierarchisch gegliedert, sodass eine breit gefächerte APT mehrere Teil-APT beinhalten kann. Die APT sollen so beschrieben werden, dass im klinischen Alltag überprüft werden kann, auf welcher Leistungsniveaustufe ein Weiterbildungsassistent in Bezug auf eine konkrete APT einzuordnen ist und welche Tätigkeiten ihm/ihr auf Basis dieser Einschätzung als Nächstes anvertraut werden können. Die Überprüfung des Leistungsniveaus kann durch direkte Beobachtung durch den Anleiter erfolgen und durch indirekte Beobachtung, wie z.B. die Bewertung von Arbeitsergebnissen oder Berichte von anderen Mitarbeitern, ergänzt werden. Die Autoren schlagen vor, für jede Facharztdisziplin idealtypisch zu beschreiben, welches Leistungsniveau in welchen Kompetenzen wann im Verlauf der Weiterbildung erreicht wird und anhand welcher APT dies dargestellt bzw. überprüft werden kann. Die Autoren weisen jedoch auch darauf hin, dass viele Kliniker den kompetenzorientierten Weiterbildungsmodellen kritisch gegenüberstehen, weil sie diese für kompliziert und umständlich halten und lediglich als akademische Konstrukte ohne praktischen Wert wahrnehmen. Es erscheint also fraglich, ob ein solches elaboriertes Konzept sich im klinischen Alltag tatsächlich durchsetzen könnte.

Grundsätzlich lässt sich feststellen, dass sich der ärztliche Berufsstand intensiv mit der Qualität der ärztlichen Weiterbildung auseinandersetzt, hier Probleme identifiziert hat und daran arbeitet, diese Probleme im Rahmen einer Reform der Musterweiterbildungsordnung anzugehen. Es bleibt abzuwarten, ob und in welcher Form diese Ziele in einer zukünftigen reformierten ärztlichen Weiterbildung realisiert werden und wie dies im klinischen Alltag umgesetzt wird.

Viele dieser Zielsetzungen erscheinen für eine mögliche Weiterbildung nach einem psychotherapeutischen Direktstudium ebenfalls relevant, und einige der für die ärztliche Weiterbildung diskutierten Veränderungen könnten daher auch für die Gestaltung der psychotherapeutischen Weiterbildung von Interesse sein.

\section{Fazit}

Wie sollte vor dem Hintergrund dieser Befunde zur psychotherapeutischen Ausbildung und zur ärztlichen Weiterbildung nun eine psychotherapeutische Gebietsweiterbildung auf hohem Qualitätsniveau aussehen? Ist es überhaupt möglich, das, was jetzt in der Psychotherapieausbildung für gute Qualität sorgt, in eine Weiter- 
bildung $\mathrm{zu}$ transferieren? Oder würden mit den grundlegenden strukturellen Merkmalen einer Weiterbildung auch die gleichen Qualitätsprobleme, wie sie in der ärztlichen Weiterbildung deutlich werden, automatisch übernommen?

Der zentrale strukturelle Unterschied zwischen Psychotherapieausbildung und Weiterbildung besteht darin, dass in der jetzigen Ausbildung der arbeitsrechtliche Status der Ausbildungsteilnehmer weitgehend ungeregelt ist. Sie sind Kunden des Ausbildungsinstitutes und bezahlen für die «Dienstleistung Ausbildung», haben in der Praktischen Tätigkeit zumeist einen Praktikantenstatus und die Therapien im Rahmen der Praktischen Ausbildung werden in der Regel auf Honorarbasis vergütet. In der Weiterbildung hingegen besteht zwischen Weiterbildungsassistenten und Weiterbildungsstätte immer ein sozialversicherungspflichtiges Arbeitsverhältnis, da Weiterbildung wesentlich durch eine angemessen vergütete Berufstätigkeit an einer Weiterbildungsstätte definiert ist. Das würde für eine psychotherapeutische Weiterbildung bedeuten, dass die verschiedenen beruflichen Einsatzorte im Verlauf der Weiterbildung, wie z.B. Klinik, Ambulanz des Institutes oder Lehrpraxis, immer auch als Weiterbildungsstätte anerkannt sein müssten und Arbeitgeber des Weiterbildungsassistenten wären. Welche Rolle würde dann das Institut spielen, wenn ein Weiterbildungsassistent z.B. gerade bei einer Klinik einen Weiterbildungsabschnitt absolviert und der Weiterbildungsbefugte der Klinik dort für die Weiterbildung zuständig ist? Um das, was an der jetzigen Ausbildung gut ist, zu erhalten, erscheint es uns hier wichtig, die Weiterbildungsordnung so zu gestalten, dass, egal in welcher Weiterbildungsstätte gerade der praktischen Berufstätigkeit nachgegangen wird, immer parallel ein Bezug zu dem Institut besteht, wo berufstätigkeitsbegleitende Qualifizierungselemente absolviert werden.

Entscheidend für die Qualität der psychotherapeutischen Qualifikation erscheinen uns daher folgende Aspekte:

- Psychotherapeutische Weiterbildung darf nicht allein aus praktischem Erfahrungserwerb bestehen. Die praktische Berufstätigkeit im Rahmen der Weiterbildung muss von Experten für das jeweilige Feld angeleitet und beaufsichtigt und kontinuierlich über die gesamte Weiterbildungszeit von Supervision, Theorie und Selbsterfahrung in nach Möglichkeit konstanten Lerngruppen begleitet werden.

- Praktische Berufstätigkeit im Rahmen der Weiterbildung sollte sowohl im ambulanten als auch im stationären Bereich absolviert werden. Es sollten umfangreiche Erfahrungen sowohl in der Akutversorgung psychisch Kranker als auch mit langen psychotherapeutischen Behandlungsverläufen gesammelt und so ein breites Spektrum an Versorgungssettings und alle relevanten Patientengruppen bzw. Störungen kennengelernt werden.

- Die bisherigen Ausbildungsinstitute für Psychotherapie verfügen über eine hohe Expertise in der Theorievermittlung und Koordinierung des Ausbildungsablaufes und gewährleisten eine hohe Strukturqualität hierfür. Diese Aufgaben sollten auch in einer Weiterbildung in der Hand der Institute bleiben. Abweichend vom Vorbild der ärztlichen Weiterbildung sollte in einer psychotherapeutischen Weiterbildungsordnung der Begriff des Weiterbildungsinstitutes als zusätzlicher Ort der Weiterbildung neben der Weiterbildungsstätte definiert werden und die Aufgaben dieses Weiterbildungsinstituts sollten beschrieben werden.

- Damit die verschiedenen Tätigkeitsabschnitte der praktischen Berufstätigkeit an den Weiterbildungsstätten und die curriculare Vermittlung von Wissen und Fertigkeiten am Weiterbildungsinstitut gut koordiniert werden und inhaltlich aufeinander bezogen sind, sollten die Weiterbildungsstätten und Weiterbildungsinstitute zur Kooperation in Form von Kooperationsverträgen oder Weiterbildungsverbünden verpflichtet werden. Eine Weiterbildung nach «Baukastenprinzip», bei der einfach nur Tätigkeitsabschnitte und Theorieblöcke gesammelt werden, die nicht aufeinander abgestimmt sind, sollte vermieden werden.

- Die im Rahmen der Weiterbildung zu erwerbenden Kompetenzen sollten beschrieben werden; der Kompetenzerwerb bzw. Kompetenzfortschritt im Rahmen der Weiterbildung sollte regelmäßig überprüft werden, sodass der Weiterbildungsverlauf flexibel an den Stand des Kompetenzerwerbs angepasst werden kann. Ein Referenzkonzept hierfür könnte das Konzept der APT darstellen.

Die Psychotherapeutenkammern wären die zentrale Instanz, die die Weiterbildung regelt, Weiterbildungsbefugnisse und Anerkennungen von Weiterbildungsstätten und Weiterbildungsinstituten erteilt und die Durchführung der Weiterbildung überwacht. Die Kammern würden in der Regel jedoch nicht selbst die Weiterbildung anbieten. Dadurch, dass jede Weiterbildungsstätte von der Psychotherapeutenkammer zugelassen werden müsste und einen von der Kammer zur Weiterbildung befugten Psychotherapeuten vorweisen müsste, wären die Probleme, die mit der Externalität der Praktischen Tätigkeit in der jetzigen Psychotherapieausbildung verbunden sind, überwunden. Der Berufsstand der Psychotherapeuten hätte somit mehr Freiheit für die Gestaltung der psychotherapeutischen Qualifizierung, aber auch mehr Verantwortung für diese Qualifizierung und den eigenen Nachwuchs.

Wir sind zuversichtlich, dass sich diese Vorstellungen im Rahmen einer (Muster-)Weiterbildungsordnung umsetzen lassen, die mit den Heilberufegesetzen bzw. Heilberufekammergesetzen der Bundesländer kompatibel ist.

Ein wesentlicher kritischer Punkt für die Umsetzbarkeit von hohen Qualitätsansprüchen in einer Weiterbildung wird jedoch letztendlich in der Frage der finanziellen Ausstattung der Weiterbildungsstrukturen liegen. Auf Basis der derzeitigen psychotherapeutischen Kassenhonorare wird es kaum möglich sein, ein angemessenes Gehalt für einen Weiterbildungsassistenten zu generieren, wenn es bei einer für einen Berufsanfänger vertretbaren Arbeitsbelastung bleiben soll und noch zeitliche Ressourcen für die curriculare Theorievermittlung und Reflexion der psychotherapeutischen Tätigkeit nötig sind.

Hier würde die von psychotherapeutischen Berufs- und Fachverbänden geforderte bessere Honorierung von Psychotherapie etwas Abhilfe schaffen, aber es wäre über die gängigen Honorare hinaus wohl eine zusätzliche Finanzierung der Weiterbildung nötig. Das Problem der Finanzierung von Weiterbildung im ambu- 
lanten Setting steht auch auf der Agenda der Kassenärztlichen Bundesvereinigung (KBV), die ein Stiftungsmodell für die Finanzierung der ambulanten Weiterbildung vorschlägt. Das Modell zur Förderung der Weiterbildung in der Allgemeinmedizin könnte hier ein Vorbild sein, aber auch ein Zuschlag zum Orientierungspunktwert für Leistungen im Rahmen der Weiterbildung, wie er vom ehemaligen KBV-Vorsitzenden Andreas Köhler [2013] vorgeschlagen wurde, wäre denkbar.

Die Forderung nach einer Finanzierung der Weiterbildung muss daher als Mindestanforderung an eine Reform in Richtung einer Direktausbildung erhoben werden. Ohne eine funktionsfähige Weiterbildungsstruktur, die finanziell so ausgestattet ist, dass ausreichend Kapazitäten für angemessen vergütete Weiterbil- dungsstellen zur Verfügung stehen, wäre die Reform nur halb umgesetzt und die Qualität der psychotherapeutischen Qualifizierung und auch der Patientenversorgung tatsächlich gefährdet. Wenn dies jedoch erreicht werden kann, sind wir zuversichtlich, dass ein hoher Qualitätsanspruch auch im Rahmen einer Weiterbildungsordnung in eigener Verantwortung des Berufsstandes der Psychotherapeuten umgesetzt werden kann.

\section{Disclosure Statement}

J.T. ist als Vorstandsreferent für den Deutschen Fachverband für Verhaltenstherapie (DVT) tätig. Alle weiteren Autoren sind Vorstandsmitglieder des DVT und in der psychotherapeutischen Aus- und Weiterbildung tätig.

\section{Literatur}

Bartmann F: TOP IV - (Muster-)Weiterbildungsordnung. Sachstand zur Novellierung. Vortrag beim 116. Deutschen Ärztetag, 2013. www.bundesaerztekammer.de/ arzt2013/media/top4/bartmann_ppt.pdf (Zugriff am 06.06.2014)

Berberat PO, Harendza S, Kadmon M: Anvertraubare professionelle Tätigkeiten - Sichtbarwerden von Kompetenz in der Weiterbildung. Positionspapier des Ausschusses Weiterbildung der Gesellschaft für Medizinische Ausbildung (GMA). GMS Z Med Ausb 2013;30: $10-19$.

Bundesärztekammer: Evaluation der Weiterbildung Ergebnisse der 2. Befragung 2011 Psychiatrie und Psychotherapie. www.bundesaerztekammer.de/downloads/ Evaluation_WB_-_Befragung_2011_Psychiatrie_ Psychotherapie.pdf, 2011a (Zugriff am 20.08.2014).

Bundesärztekammer: Evaluation der Weiterbildung - Ergebnisse der zweiten Befragung 2011 Psychosomatische Medizin und Psychotherapie. www.bundesaerztekammer.de/downloads/Evaluation_WB_-_Befragung 2011_PsychosomatischeMed_Psychotherapie.pdf, 2011b (Zugriff am 20.08.2014).

Bundesärztekammer: Evaluation der Weiterbildung - Ergebnisse der WBB-Befragung 2011 Gebiete Kinderund Jugendpsychiatrie und -psychotherapie; Psychiatrie und Psychotherapie; Psychosomatische Medizin und Psychotherapie. www.bundesaerztekammer.de/ downloads/EvaluationWB2011PPP120613.pdf, 2011c (Zugriff am 20.08.2014).

Bundesärztekammer: Kompetenzbasiert und flexibel Reform der (Muster-)Weiterbildungsordnung. BÄKground Spezial - Hintergrundinformationen für Journalisten zum 116. Deutschen Ärztetag, 2013. www.bundesaerztekammer.de/downloads/BAeKground_ Spezial Weiterbildung.pdf (Zugriff am 20.08.2014).

Bundesärztekammer, Consumer Behaviour Group ETH Zürich: Ergebnisse der Evaluation der Weiterbildung 2. Befragungsrunde 2011 Bundesrapport 2011. $w w w$. bundesaerztekammer.de/downloads/Bundesrapport 2011.pdf, 2011 (Zugriff am 20.08.2014).
Bundespsychotherapeutenkammer: Kompetenzen für den Psychotherapeutenberuf in Studium und Aus-/Weiterbildung - Entwurf der AG des Länderrates und BPtKVorstands (Fassung vom 06.05.2014). www.bptk.de/ uploads/media/20141118_kompetenzprofil_stand_ 06052014.pdf, 2014 (Zugriff am 17.11.2014).

David D, Euteneier A, Fischer M, et al.: Die Zukunft der ärztlichen Weiterbildung in Deutschland - Positionspapier des Ausschusses Weiterbildung der Gesellschaft für Medizinische Ausbildung (GMA). GMS Z Med Ausb 2013;2:1-6.

Ericsson K: Deliberate practice and acquisition of expert performance: a general overview. Acad Emerg Med 2008;11:988-1094.

Fliegel S: Direktausbildung Psychotherapie - Ein Weg mit fatalen Konsequenzen. www.gesundheitspolitik.de/ wp-content/uploads/2013/04/Direktausbildung-Psychotherapie-Ein-Weg-mit-fatalen-Konsequenzen.pdf, 2013 (Zugriff am 30.01.2014).

Gleiniger JW: Basal oder dual? Ordnungspolitische Rechtfertigung einer Direktausbildung der Psychotherapeuten auf dem Prüfstand. VPP 2013;2:493-517 (Sonderdruck).

KBV (Kassenärztliche Bundesvereinigung): Weiterbildung im ambulanten Bereich fördern. www.kbv.de/html/ 4475_9508.php, 2014 (Zugriff am 28.10.2014).

Köhler A: Bedeutung der Weiterbildung für den Arzt- und Psychotherapeutenberuf. Psychotherapie Aktuell 2013; 4:9-11.

Körner J: Plädoyer für eine Direktausbildung zum Psychotherapeuten. Forum Psychoanal 2013;2:235-257.

Lubisch B: Könnte so die Direktausbildung aussehen? Eine Skizze. Psychotherapie Aktuell 2012;3:28-31.

Marburger Bund: MB-Monitor 2014: Ärztliche Weiterbildung - Zusammenfassung der Ergebnisse. $w w w$. marburger-bund.de/sites/default/files/artikel/downloads/ 2014/mehrheit-der-jungen-aerztinnen-und-aerzte-will-imkrankenhaus-bleiben/zusammenfassung-mb-monitor2014.pdf, 2014 (Zugriff am 05.06.2014).

Richter R: Das Berufsbild der zukünftigen Psychotherapeuten. Psychotherapie Aktuell 2013;4:13-15.
Rief W, Fydrich T, Margraf J, Schulte D: Modellvorschlag Direktausbildung Psychotherapie (Version 3). Berlin, Kommission Psychologie und Psychotherapie, Deutsche Gesellschaft für Psychologie, 2012a. www.dgps. del_download/2012/Direktausbildung_Psychotherapie_ Vers_3_2012-04-02.pdf (Zugriff am 20.11.2014).

Rief W, Schulte D, Vogel H, Kuhr A: Pro und Kontra Direktausbildung Psychotherapie. Verhaltenstherapie 2012b;22:56-63.

Sachse R, Fasbender J, Hammelstein P: Wie Psychotherapie-Ausbildung sein sollte - Eine psychologische Analyse didaktischer Erfordernisse; in Sulz SKD (ed): Psychotherapie ist mehr als Wissenschaft - Ist hervorragendes Expertentum durch die Reform gefährdet? München, CIP-Medien, 2014, pp 15-37.

Skovholt T, Ronnestad MH, Jennings L: Searching for expertise in counseling, psychotherapy, and professional psychology. Educ Psychol Rev 1997;4:361-369.

Strauß B, Barnow S, Brähler E, Fegert J, Fliegel S, Freyberger HJ, Goldbeck L, Leuzinger-Bohleber M, Willutzki U: Forschungsgutachten zur Ausbildung von Psychologischen PsychotherapeutInnen und von Kinder- und JugendlichenpsychotherapeutInnen, im Auftrag des Bundesgesundheitsministeriums. Jena, 2009.

Ströhm W, Schweiger U, Tripp J: Konzept einer Weiterbildung nach einer Direktausbildung in Psychotherapie. Psychotherapeutenjournal 2013;3:262-268.

Sulz SKD: Weiterbildung nach der dualen Direktausbildung in Psychotherapie - Ein Konzept zur Gestaltung der Zukunft der Psychotherapie. Psychotherapie 2013; 2:237-254.

Sulz SKD, Hoenes A: Wieso das Facharztweiterbildungsmodell ungeeignet für die Psychotherapeutenausbildung ist; in Sulz SKD (ed): Psychotherapie ist mehr als Wissenschaft - Ist hervorragendes Expertentum durch die Reform gefährdet? München, CIP-Medien, 2014, pp 290-305.

Tripp J: Konzept einer Weiterbildung nach einer Direktausbildung in Psychotherapie. Psychotherapie Aktuell 2013;4:6-8.

Weck F: Psychotherapeutische Kompetenzen - Theorie, Erfassung, Förderung. Berlin, Springer, 2013. 